\title{
Research on Refrigeration Equipment for Drinks Based on Semiconductor Refrigeration Technology
}

\author{
Li Jinliang \\ Zibo Vocational Institute Industrial Engineering and Operations Department, China \\ alj1522@163.com
}

Keywords: Staged cooling; Semiconductor refrigeration; Fluent; Refrigerating capacity

\begin{abstract}
In order to meet the demand of personalized cold drinks in the market, the refrigeration technology was analyzed and researched, and the scheme of staged cooling was established. Based on the principle of semiconductor refrigeration technology, which the semiconductor refrigeration device controlled by the PID , fluent software was used for simulation of semiconductor refrigeration heat and radiator end, then radiating end of the physical parameter was optimized. Through the experiment for staged cooling device, the main performance indexes of the equipment were tested, the simulation results were verified, and the work efficiency of the small refrigeration unit was improved.
\end{abstract}

\section{Introduction}

With the improvement of the living standard of the national family, in order to satisfy the different needs for cold drinks, it takes a long time to freeze in the refrigerator (30 minutes or more) to reach the drinking requirement, especially in scorching summer. All drinks are consumed at the same temperature, it`s unable to meet the personalized needs of people, and the cooling time is long. At present, the usual refrigeration methods in refrigeration equipment are as follows: ammonia liquid cooling, thermoelectric cooling, magnetic refrigeration, electrochemical refrigeration, thermoacoustic refrigeration, pulse control cooling and so on, specific analysis of the above refrigeration technology, high cost, energy consumption, high risk, not suitable for use in family, especially the equipment is inconvenient to carry.

Based on the above analysis, at present, semiconductor refrigeration technology is used to solve this problem. This technology occupies a small space, cooling rapidly, the temperature can be adjusted precisely, no refrigeration required and other advantages. Semiconductor refrigeration is also called thermoelectric refrigeration; it is the thermoelectric effect of semiconductor materials. When there is no external magnetic field, the thermoelectric effect mainly includes See beck effect, Peltier effect, Thomson effect, Joule effect and Fourier effect. The working principle is shown in figure 1. Study how to achieve a smaller temperature difference between hot and cold ends, control of current or voltage to achieve accurate control of cooling output is the key to achieve rapid refrigeration.

\section{Analysis of Semiconductor Refrigeration Conditions}

To maximize the efficiency of the semiconductor refrigeration element, the temperature difference between the hot and cold ends must be reduced. The heat dissipation modes at the hot end include air cooling, water cooling, heat pipe cooling heat dissipation and so on, at the same time; cold ends also need to be considering for accelerated transmission of cold capacity.

To analysis the heat radiation condition, determine the best way to heat dissipation, change the current value on this basis, achieve the best coefficient of refrigeration, As the shown in figure 2, a physical model of the thermal end is established. 


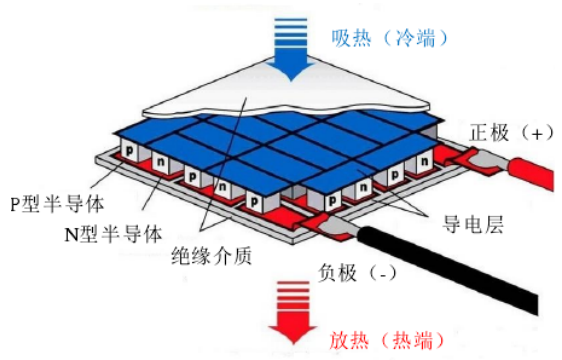

Fig.1 schematic diagram of semiconductor

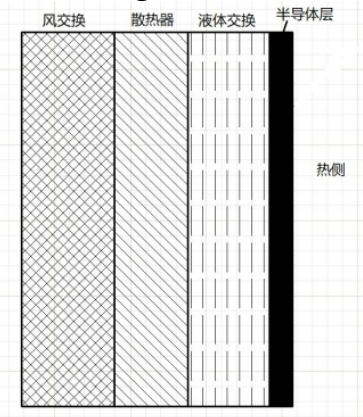

Fig.2 physical model of semiconductor cooler refrigeration chip

Analysis in ideal state, just consider the Joule effect from the inside and the Fuliye effect the lose of these two effects, the loss of the heat resistance from external heat transfer is not considered, let's say it's an externally reversible semiconductor refrigeration system. At the same time Thomson effect as the secondary effect, it's value is small compared with the Fouier transform, it can be ignored in engineering or design calculation. Based on the low of conservation of energy the coefficient of refrigeration is obtained:

the coefficient of refrigeration:

$$
\varepsilon=\frac{Q_{1}}{P}=\frac{\alpha I T_{C} \frac{I^{2} R}{2} K \Delta T}{\alpha I \Delta T+I^{2} R}
$$

$T_{c}$ in the formula is semiconductor cold end absolute temperature, $\Delta T$ is the cold and hot end temperature difference, $\mathrm{R}$ is the semiconductor resistance, $\mathrm{K}$ is a semiconductor thermal conductor, I is passing current, $T_{h}$ is the absolute temperature of semiconductor heat terminal.

The main limiting conditions of semiconductor refrigeration are maximum cooling efficiency $\varepsilon_{\max }$ and maximum refrigerating capacity $Q_{\max }$. The former can make the most efficient use of electric energy in the process of refrigeration; the latter is for maximum temperature reduction. It is difficult to maintain a stable under certain limiting conditions, it is carried out in the working current region of the two working conditions, and the design working condition must take into account the refrigerating and cooling efficiency.

The Refrigeration Efficiency. By the formula 1 we can known, the cooling coefficient is related to the current and the cold end temperature, by taking the derivative of the current, can get the values of the coefficient of refrigeration $\varepsilon$, can obtain the maximum refrigeration factor. When the derivate value is 0 , take the extreme, the expression of the current in the maximum cooling coefficient is obtained as follow:

When $T_{C}, T_{h}$ are deternined, let $\partial_{\varepsilon} / \partial_{\mathrm{I}}=0$, by 1 the optimal current value in this case: $I_{\varepsilon}=\frac{\alpha \Delta T}{R(M-1)}$.

Maximum cooling efficiency:

$$
\varepsilon_{\max }=\frac{M T_{c}-T_{h}}{\Delta T(M+1)}=\frac{T_{c}}{T_{h}-T_{c}} \frac{M-T_{h} / T_{c}}{M+1}
$$

The Maximum Refrigerating Capacity. Multiple effects exist in the process of semiconductor refrigeration, it`s not that the more power you put in, the more cold you get, because the heat end of the heat also increases with the increases of power, there will be a decrease in the amount of input 
power. According to the application requirements, input different current to the semiconductor refrigerator, in order to achieve the best effect.

Let $\partial Q_{c} / \partial I=0$, take the derivative with respect to the refrigerating quality, obtained the maximum amount of current corresponding to the maximum refrigeration capacity: $I_{Q}=\frac{\alpha T_{c}}{R}$

The maximum refrigerating capacity:

$$
Q_{\max }=\frac{\left(\alpha T_{c}\right)^{2}}{2 R}-K \Delta T=\frac{\alpha^{2}}{R}\left(\frac{T_{c}^{2}}{2}-\frac{\Delta T}{Z}\right)
$$

The refrigeration efficiency under maximum refrigeration condition:

$$
\varepsilon=\frac{1}{2 T_{h}}\left(T_{c}-\frac{2 \Delta T}{Z T_{c}}\right)
$$

Under maximum cooling output, because of the input current is large, the heat at the end of the cooling plate is relatively large, the effect of the Fourier is also greater, so the overall cooling efficiency is very good in the case of maximum refrigerating, otherwise, it`s lower. In $\varepsilon_{\max }$ condition, the refrigerating quantity is much smaller than the theory $Q_{\max }$, the best current in $I_{Q}$ and $I_{\varepsilon}$ to take.

In beverage separation refrigeration equipment, need to study input and output, make $\varepsilon$ as far as possible big. Analysis in the formula of refrigerating quantity and coefficient of refrigeration can obtained, it's size is related to the temperature difference $\Delta T$ between the cold and hot end, the greater the value, the greater the heat conduction between the cold and hot end, the greater the loss of cold energy. In order to improve the efficiency of semiconductor refrigeration, cold and hot end heat exchangers need a stronger heat exchange capacity, make the temperature of the hot end as low as possible, make the temperature of the cold end get higher, reduce the $\Delta T$ values.

\section{Model Creation And Simulation Mode}

According to the previous analysis of refrigeration coefficient and refrigerating capacity, the cooling end of the beverage was simulated and analyzed, building simulation model, obtained the optimal current value, come true the optimal refrigeration capacity, achieve rapid grading refrigeration. The equipment choose TEC1-12704 as semiconductor refrigeration components, see figure 3. It has high strength, thermal conductivity and so on advantages, a total 16 semiconductors are used in parallel. The heat dissipation of the equipment is the heat flow, plate of heat dissipation combination, use Fluent software to the simulation, optimize the inlet velocity and the number of fins and dimensions.

Set up Global Physical Model. At first set up model in software at first, and grid partitioning, set boundary conditions and so on.
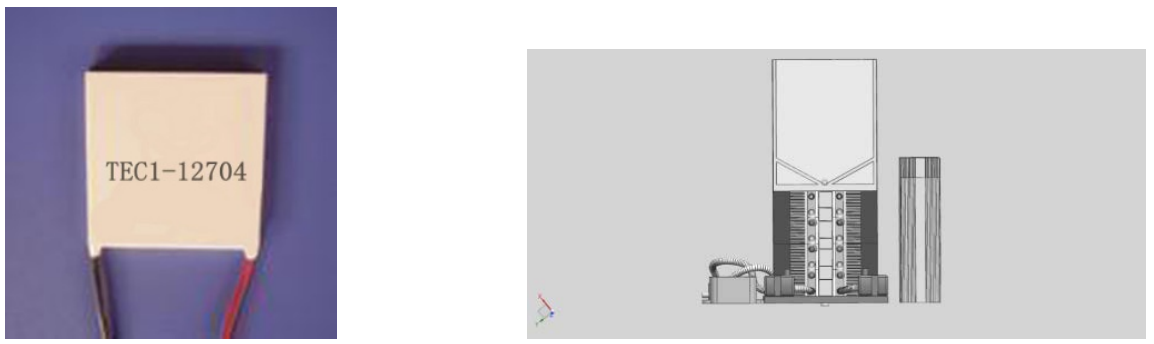

Fig.3 refrigerant chip diagram refrigeration Fig.4 three dimensional model of staged

The quality of grid(Interval Size)is $1 \mathrm{~mm}$, the number of grids after partitioning is 498213 . The model consist of three regions, radiator area, gas flow region, liquid flow region. The radiator is made of aluminum, the material of the liquid flow region is recycled water, the material of the gas flow region is air, the thermal conductivity is $0.0242 \mathrm{~W} / \mathrm{m} \cdot \mathrm{k}$, the viscosity coefficient is $1.7894 \mathrm{e}-05$. Set the inlet velocity of water and gas separately: $0.3 \mathrm{~m} / \mathrm{s} 、 5 \mathrm{~m} / \mathrm{s}$. The boundary conditions are defined by fluid solid coupling, heat flow at the wall of heat source is set to $90000 \mathrm{~W} / \mathrm{m}^{2}$.

Analysis the Simulation Results. Fluent solution methods, separation and solution is to solve each of the governing equations, the nonlinear governing equations need to be iterated to conform to the convergence conditions. 
Analysis the Velocity Field. Seen from the figure 5 we can know, at the center of the radiator bottom, the velocity is close to 0 , the maximum velocity is near the exit, it directly affects the cooling effect.

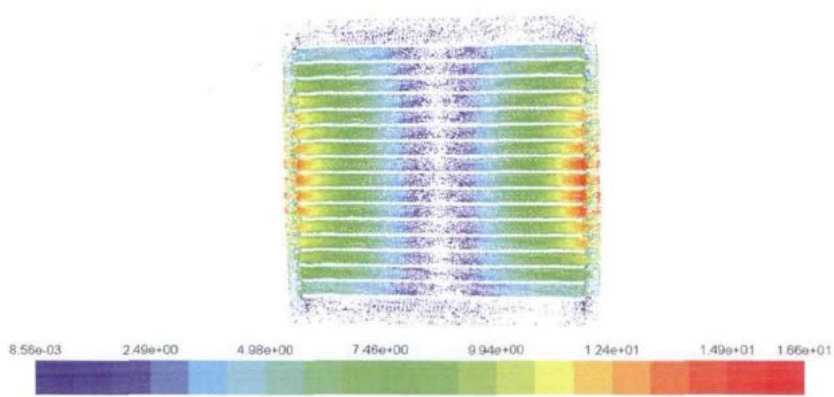

Fig.5 central velocity distribution at bottom of radiator

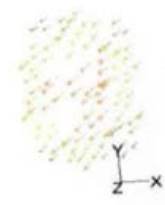

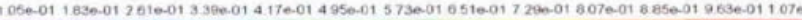

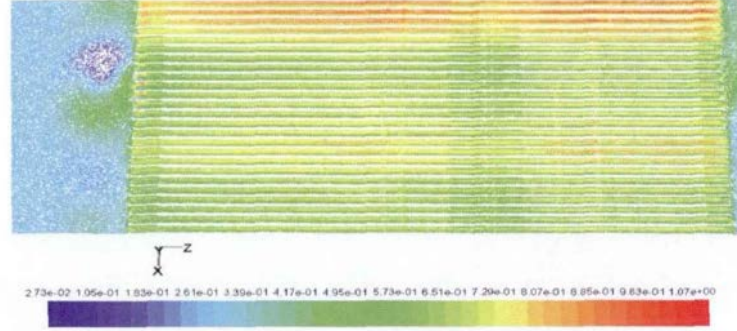

Fig.7 central velocity distribution of liquid pipeline

Fig.6 velocity distribution at liquid inlet

f the liquid pipeline, the flow velocity is the highest, maximum up to $1.07 \mathrm{~m} / \mathrm{s}$, pipe wall accessories have the lowest speed. In the pipeline, the liquid does not flow into each channel at the same speed.

Analysis the Temperature Field. In the model, heat flux at heat end is set to $90000 \mathrm{~W} / \mathrm{m}^{2}$, the temperature of the heat source surface can directly see the heat radiation effect of the radiator.

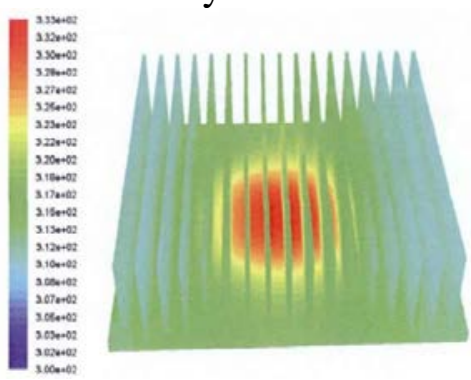

Fig.8 the overall distribution of radiators

You can see it from the figure above, the temperature distribution is not uniform, the intermediate temperature is above the edge, one reason is the flow in the middle is low, another reason is the thermal conductivity of the radiator is low, thermal resistance has a negative effect on the diffusion of heat.

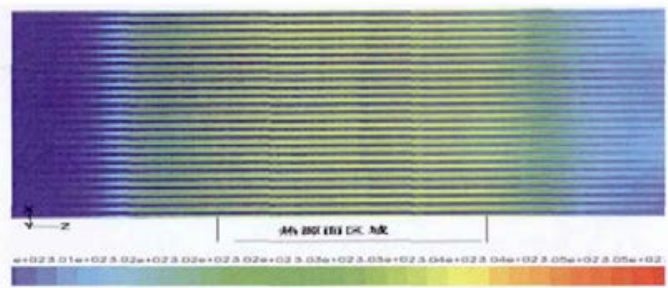

Fig.9 temperature distribution at the entrance of the liquid

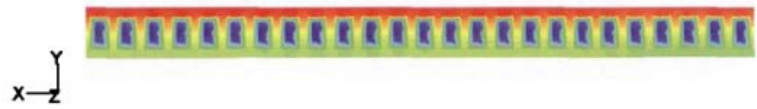

$3.00 \theta+02300 \theta+023.01 \theta+023.01 \theta+023.02 \theta+023.02 \theta+023.02 \theta+023.03 \theta+023.03 \theta+023.04 \theta+023.04 \theta+023.05 \theta+023.05 e+02305 \theta+16$

Fig.10 temperature distrubution of liquid piping cente 
Analysis the figure9.10, when the water flows through the reservoir, there is a marked increased in temperature, this indicates that this way there is a significant convective heat transfer coefficient, after water flows out of the pipe, the heat spreads evenly, the water temperature uniform distribution, it s hotter than the entrance, the liquid in the pipe near the heat source surface is higher than the temperature near the center. Because the water is in circulation, and there is good convection in the air, the heat dissipation is good, it is no need to analyze the temperature in the air.

\section{Experimental Study on Heat Sink of Semiconductor Refrigeration}

Experimental equipment mainly include: semiconductor refrigerator, cold and hot end ribs, circulating water channel, water pump, the cold end plate, axial flow fan, thermal conductive silicone, temperature sensor, temperature acquisition instrument, computer, heat preservation material, DC power supply and so on.

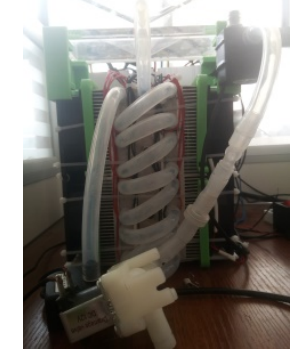

Fig.11 grading refrigeration laboratory equipment

Main experimental procedure: based on above equipment, set up the experiment device as shown in figure 11, install temperature sensor in it's refrigerator space, the change curve of heat exchange and fin heat dissipation is obtained by using the temperature of the computer every two minutes.

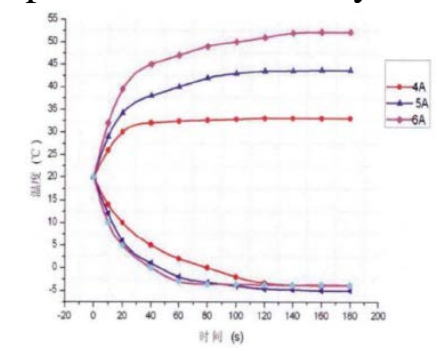

Fig.12 time variation curve of plate heat dissipation under different input power

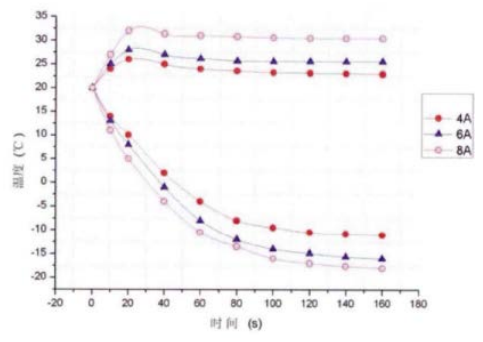

Fig.13 Curves of fluid temperature versus time under different input power

Through, if the hot end uses a single sink, this heat dissipation method has a large temperature difference, the larger the current input is, the greater the change in temperature. So in the case of the same current value, select two combinations heat dissipation the hot end, the ratio of current and refrigeration can be realized effectively, improve cooling efficiency.

\section{Conclusion}

The heat dissipation mode of semiconductor refrigerator is studied in this paper, on the basis of simulation analysis of two different heat dissipation modes, a test device for the study of heat dissipation of fractional refrigeration equipment was set up, and the device comprises a fin fan a fluid radiator. Get under a different current; two heat sinks are used in combination, best heat dissipation, it reduces the temperature difference, and the next step is to use PID control to achieve hierarchical control temperature laid the foundation.

\section{Acknowledgment}

In this paper, the research was sponsored by A Project of Shandong Province Higher Educational Science and Technology Program, (Project No. J14LB63). 


\section{Reference}

[1]Cao Hui, He Bo, Liu Minghui, Chen Jinliang, Application of fuzzy control in temperature control system of reheating furnace [J], Modern manufacturing engineering, 2010, (05):137-141

[2]Jiang Jing. Constant temperature box temperature regulation and parameter setting method [J]. Instrument user, 2009, Vol.16 (03):110-111.

[3]Riffat S B, Ma Xiaoli. Thermoelectrics: a review of present and potential application. Applied Thermal Engineering, 2003,23(8):913-935.

[4]Dai Hong-Yuan, Wen Hong, Yu Na, Cao Zhi-Gao, Experimental study of heat pipe cooling semiconductor refrigeration system, [J]. journal of Nanchang university, 2012（01）.

[5]Hu Guang-Xin, Study on heat dissipation characteristics and application of fin and microchannel heat sink, [D]. University of Electronic, 2010. 